Revista de

Contabilidade e

Organizações

www.rco.usp.br
DOI: http://dx.doi.org/10.11606/rco.v9i24.81136

Journal of

Accounting and

Organizations

\title{
Social disclosure e retornos anormais: um estudo de eventos em empresas brasileiras abertas no período de 2005 a 2012
}

\author{
Rodrigo de Souza Gonçalves ${ }^{\mathrm{a}}$, Natália de Menezes Barbosa ${ }^{\mathrm{a}}$, Caio Rodrigues Barroso ${ }^{\mathrm{a}}$ \\ Otávio Ribeiro De Medeiros ${ }^{\mathrm{a}}$
}

${ }^{a}$ Universidade de Brasília

\section{Informações do Artigo}

Histórico do Artigo

Recebido: 03 de junho de 2014

Aceito: 05 de setembro de 2015

Palavras chave:

Social Disclosure

Estudo de Evento.

Reputação Corporativa

Eficiência de Mercado

Retorno Anormal

\begin{abstract}
Resumo
O presente estudo investiga se o evento da divulgação da informação social é determinante para melhoria do retorno acionário das empresas brasileiras de capital aberto. Foram analisadas 49 empresas brasileiras listadas na BM\&FBovespa, que divulgaram relatórios de responsabilidade social no período de 2005 a 2012, totalizando 254 observações. Os níveis de social disclosure foram mensurados por um índice (ISD), baseado em Ramanathan (1976), Haydel (1989) e Hammond e Miles (2004), que avalia as informações através de 13 indicadores. Os resultados encontrados apontaram que, para as empresas de maior ISD há uma tendência de maiores retornos, sobretudo imediatamente após a divulgação da informação. Os referidos resultados foram corroborados ao nível de significância de $5 \%$, indicando que empresas com maiores níveis de social disclosure tendem a apresentar retornos anormais acumulados positivos e estatisticamente significantes.
\end{abstract}

Copyright (C) 2015 FEA-RP/USP. Todos os direitos reservados

\section{INTRODUÇÃO}

A decisão de realizar investimentos em determinado ativo passa pela análise do risco ao qual o investidor estará exposto, considerando o retorno que ele espera obter no investimento realizado. Nesse sentido, a busca por informações acerca do ativo é um dos passos necessários para a realização de qualquer investimento. No caso do mercado de capitais, uma das principais fontes de informações acerca das empresas são os relatórios contábeis, que são compostos por informações obrigatórias e voluntárias e geralmente consolidados em divulgações anuais.

Atualmente, em razão do desenvolvimento do mercado de capitais brasileiro, que, entre outros fatores, está associado à entrada de recursos estrangeiros, as empresas, com o propósito de tornarem-se mais transparentes, têm buscado meios de divulgar uma maior quantidade de informações voluntárias, entre as quais as relacionadas à responsabilidade social corporativa (social disclosure - SD). O SD deve servir como um instrumento de relacionamento entre empresa e sociedade que pode, no longo prazo reduzir o risco reputacional, trazendo melhorias para a imagem da organização e consequentemente contribuir para a captação de recursos mais atrativos para a empresa (MACHADO FILHO; ZYLBERSZTAJN, 2003; DHALIWAL et. al., 2011).

Por outro lado, deve-se considerar que, embora se espere, de acordo com a teoria dos stakeholders (JENSEN, 2001), que haja valorização da empresa diante da obtenção de uma melhor imagem corporativa, ocorrem gastos adicionais para se efetivar tal ação, o que, por sua vez, pode acabar suprimindo os ganhos da empresa (BRIGHAM; GAPENSKI; EHRHARD, 2001) e ainda gerar desconfiança, a ponto de ocorrer como afirmam Carr e Outhwaite (2009) acerca da ocorrência de "corrupção nas práticas de responsabilidade social corporativa". Apesar disso, pesquisas envolvendo mercados desenvolvidos como as de Poddi e Vergalli (2009), Orlitzky e Benjamin (2001), Dhaliwal et. al. (2011), Reverte (2012) e Ng e Rezaee (2012) corroboram o fato de que as informações sociais contribuem para a redução do custo de captação de recursos.

Autor Correspondente: Tel (61) 31070798

E-mail : rgoncalves@unb.br (R. S. Gonçalves); barbosa.natalia@yahoo.com.br (N. M. Barbosa); caiorbarroso@gmail.com (C. R. Barros);

otavio@unb.br (O. R. Medeiros)

Universidade de Brasília - Fundação Universidade de Brasília, Cca Departamento de Ciências Contábeis e Atuariais

Campus Universitário Darcy Ribeiro - Asa Norte - 70910-900 - Brasilia, DF - Brasil 
Contudo, quando se trata de mercados emergentes os resultados ainda são controversos. Por exemplo, Rover e Murcia (2010), com uma amostra de empresas brasileiras e Gana e Dakhlaoui (2011) com uma amostra de empresas tunisinas, concluíram que o custo de capital próprio é mais elevado nas empresas que possuem maior nível de evidenciação. Entretanto, os resultados de Gonçalves et. al. (2013) vão ao encontro da hipótese de que a relação entre nível de social disclosure e custo de capital próprio no mercado brasileiro é negativa, de modo semelhante aos resultados encontrados para mercados desenvolvidos. Ainda, quando se trata do mercado financeiro no Brasil, outro aspecto que permeia essa discussão refere-se ao momento em que o mercado acionário precifica os ativos com base em novas informações, isto é, se tal mercado pode ser considerado eficiente, conforme proposto por Fama (1970).

Assim, a presente pesquisa tem por objetivo investigar se o evento da divulgação da informação social (SD) é determinante para melhoria do retorno acionário das empresas brasileiras abertas. Os objetivos específicos são: a) analisar qualitativamente e quantitativamente o social disclosure das empresas brasileiras de capital aberto; b) identificar o momento (data) em que tais informações foram disponibilizadas; c) quantificar o retorno anormal das ações; d) avaliar, a partir de uma janela de evento, o efeito provocado pelo social disclosure no retorno das ações.

Para atingir tais objetivos, foi utilizada a metodologia de estudo de eventos. Sob esse prisma, a presente pesquisa possui relevância à medida que se busca analisar a reação do mercado financeiro diante da divulgação de informações voluntárias. Esse estudo avança em relação às pesquisas anteriores (SILVA; COELHO; LUZ, 2008; CAPARELLI, 2010), uma vez que estas centraram sua análise na adesão da empresa ao Índice de Sustentabilidade Empresarial (ISE) da BMF\&Bovespa. Nesta pesquisa, não somente foi avaliada a informação divulgada em janelas específicas, isto é, de acordo com a data da divulgação em que cada empresa publicou o relatório de sustentabilidade empresarial, como também o nível de evidenciação da mesma.

\section{REFERENCIAL TEÓRICO}

\subsection{Ações de responsabilidade social corporativa e suas motivações}

A busca das empresas em alinharem seus objetivos de modo a causar o menor impacto possível na sociedade, fez com que desde o final da década de 1960 e início da década seguinte surgissem pesquisas na área contábil acerca de sua contribuição na área social (RAMANATHAN, 1976). Segundo Deegan (2002), apesar de as pesquisas nessa área terem se iniciado naquele período, a quantidade de pesquisas acerca da divulgação das ações de responsabilidade social entrou em grande crescimento a partir de 1990, em razão do interesse das organizações de utilizarem seus relatórios como instrumento de relacionamento com a sociedade. Assim, por intermédio desse mecanismo, verifica-se a busca pela legitimação empresarial.

Desse modo, há pelo menos duas motivações principais para as empresas adotarem práticas de responsabilidade social: algumas por acreditarem que um bom relacionamento com seus acionistas pode atrair maiores retornos financeiros, em função da transparência de suas ações (RICHARDSON; WELKER; HUTCHINSON, 1999; ORLITZKY, BENJAMIN, 2001; BROWN; HELLAND; SMITH, 2006); outras praticam e divulgam ações de responsabilidade social para atenderem a certos ideais e expectativas dos acionistas acerca da forma pela qual as operações da empresa devem ser conduzidas (BRANCO; RODRIGUES, 2008).

Como consequência, McWiliams, Siegel e Teoh (1999) apontam duas possibilidades de impactos causados por atividades socialmente responsáveis nas empresas. Primeiramente, se as atividades socialmente responsáveis fizerem com que a empresa deixe de realizar investimentos rentáveis, ou se a sua implementação for cara e não trouxer benefícios inerentes aos acionistas, essas atividades irão diminuir o valor da empresa, indo ao encontro dos resultados de Sternberg (1999), Hillman e Keim (2001) e Carr e Outhwaite (2009).

Em outro sentido, as atividades de responsabilidade social podem aumentar o valor da empresa, nos seguintes casos: a) se for exigida e valorizada pelos investidores (IOANNOU; SERAFIM, 2009; GOLLIER; POUGET, 2014); b) se for aumentar a produtividade da empresa através da satisfação dos empregados (PORTER; KRAEMER, 2002); c) se ocorrer aumento de preço de seus ativos no mercado acionário (CHENG; IOANNOU; SERAFEIM, 2014); e d) caso reduza os custos com clientes inadimplentes. Finalmente, as ações de responsabilidade social corporativa podem não provocar efeitos no valor da empresa se os custos e benefícios se anularem (SAIIA; CARROLL; BUCHHOLTZ, 2003; AHMED et. al., 2014). Diante desses aspectos, quando a empresa aloca recursos empresariais em atividades sociais, há necessidade, quer por obrigatoriedade legal ou não, de sua divulgação, realizada principalmente por meio dos relatórios sociais.

Para Arcay e Vázquez (2005), a política de divulgação de ações voluntárias aponta como ideia básica 
que estas são dependentes de um trade off entre os custos da evidenciação das informações e os retornos. Nesse sentido, segundo Branco e Rodrigues (2008), há evidências de que as empresas têm preferência pelo relatório anual como meio de divulgação de suas ações de responsabilidade social. Apesar de tais aspectos, Patten (1992) e Ahmed et. al. (2014), apontam que não há relação significante entre a quantidade de informações divulgadas e o desempenho social com os indicadores de rentabilidade empresarial. Para Patten (1992), as informações sociais são mais monitoradas por agentes da sociedade do que propriamente pelo mercado financeiro.

Contudo, ainda na década de 1990, Herremans, Akathaporn e McInnes (1993) encontraram evidências de que na década de 1980 nos Estados Unidos, as grandes empresas que se preocupavam em manter uma boa reputação por meio de ações de responsabilidade social obtiveram melhores resultados ou retornos quando comparadas às empresas que não adotavam essas mesmas práticas. Corroborando essa evidência, Rockness e Williams (1988) e Epstein e Freedman (1994) revelam que os investidores buscam analisar essas informações para tomar suas decisões de investimentos, enquanto que Ioannou e Serafeim (2010) constatam que empresas socialmente responsáveis recebem melhores avaliações dos analistas em razão de sua estratégia de longo prazo.

Em princípio, os resultados apontados pelas pesquisas partem da ideia de que empresas consideradas como possuidoras de melhor reputação perante os investidores venham a inspirar mais confiança e, assim, a receber mais investimentos, pois os investidores assumiriam menor risco ao aplicar seu capital nestas empresas (RICHARDSON; WELKER; HUTCHINSON, 1999; DHALIWALL et. al., 2011; JO; HARJOTO, 2014).

Para Anderson e Frankle (1980) e Dhaliwall et. al. (2011) dependendo do impacto que essa informação cause no mercado acionário, o ônus da responsabilidade social pode passar a representar uma vantagem para a empresa, e no caso do social disclosure, este pode estar associado a um retorno positivo no mercado de ações. Para Stocken (2000), Heal (2004) e Jo e Harjoto (2014), esse posicionamento justifica-se em razão de o investidor, ao receber uma informação adicional e não obrigatória para a análise da empresa, passa a analisá-la sob uma perspectiva diferenciada em relação às demais.

Há, no entanto, autores como Waddock e Graves (1997) e Mackey et. al. (2007) que defendem que esses custos não superam os benefícios para as empresas, pois reduzem os lucros e a riqueza dos acionistas, ao passo que tais investimentos não maximizam o valor da empresa. Além disso, pode ocorrer que investimentos em ações de responsabilidade social afastem investidores, considerando-se que esses podem não possuir preocupações ou interesses em relação a ações sociais, ou seja, podem estar interessados apenas em obter um maior retorno sobre capital investido (FRIEDMAN, 1970; STERNBERG, 1999).

\subsection{Social disclosure e o retorno no mercado de capitais}

A discussão acerca da repercussão da informação, incluindo a de natureza contábil, no mercado financeiro é controversa, desde as primeiras pesquisas de natureza positiva, tal como o estudo seminal de Ball e Brown (1968). No caso das informações sociais, a busca por respostas tem início na década de 1980, com pesquisas que conjugaram indicadores econômico-financeiros de retorno com a quantidade de informações divulgadas (ANDERSON; FRANKLE, 1980; WADDOCK; GRAVES, 1997).

Posteriormente, surgem pesquisas que buscam conjugar as informações sociais divulgadas com indicadores de mercado, como custo de capital próprio, valor econômico adicionado (EVA), retorno anormal, Q-Tobin, entre outros, como mostram Richardson e Welker (2001), Hillman e Keim (2001), Poddi e Vergalli (2009), Dhaliwall et. al. (2011), Gonçalves et. al. (2013), Lourenço et. al. (2014), entre outros.

As pesquisas mais recentes, a partir de 2000, quando realizadas em mercados desenvolvidos, apontam uma tendência de que o social disclosure pode repercutir de forma positiva, à medida que os resultados encontrados são de que, para tais empresas, há uma relação negativa para com o custo de captação de recursos (ORLITZKY; BENJAMIN, 2001; DHALIWAL et. al., 2011; GHOUL et. al., 2011; REVERTE, 2012; NG, REZAEE, 2012; SERVAES; TAMAYO, 2013; CAHAN et. al., 2014), ou ainda um maior retorno econômico (PODDI, VERGALLI, 2009).

A partir dos estudos ora mencionados, os fatores associados ao menor custo de captação de recursos devem-se ao fato de que a maior divulgação implica em maior transparência das ações organizacionais, que, por sua vez, aumenta a liquidez das ações, reduz o risco e, consequentemente o custo de capital. Além disso, para Ghoul et. al. (2011) e Cahan et. al. (2014), as empresas com essas características, ao terem uma maior cobertura por parte da imprensa especializada, passam a receber avaliações positivas, o que por sua vez contribui para melhoria de sua imagem reputacional.

No estudo de Cahan et. al. (2014) foi identificado que as empresas, no período de 2003 a 2011, com melhores indicadores de desempenho sociais, recebem da mídia uma cobertura com informações favoráveis, o 
que por sua vez passou a repercutir no custo de capital (mensurado pelo modelo de Ohlson) e no valor da empresa, neste caso mensurado pelo Q de Tobin. Embora, considerando os pressupostos e resultados ora mencionados, estão sustentados na hipótese de eficiência de mercado proposta por Fama (1970), quando os estudos foram realizados em mercados emergentes, como no caso brasileiro, os resultados são controversos.

No estudo de Silva e Quelhas (2006) sobre a relação entre a sustentabilidade e o custo de capital próprio, foi constatada uma relação negativa entre a adesão ao índice de sustentabilidade empresarial e o referido indicador (custo de capital próprio mensurado pelo CAPM), apontando-se que há uma possível melhora no valor econômico da empresa diante de tal evento. Contudo, os estudos de Rover e Murcia (2010) e de Gana e Dakhlaoui (2011), concluíram que o custo de capital próprio é mais elevado para empresas com maior nível de evidenciação, incluindo as de natureza social. No caso do estudo de Rover e Murcia (2010) foram analisadas as 100 maiores companhias brasileiras não financeiras no período de 2006 a 2008, sendo as informações sociais avaliadas por meio de uma escala dicotômica, a partir das dimensões: informações financeiras sociais; produtos/ serviços e colaboradores.

Entretanto, em estudo realizado por Gonçalves et. al. (2013), em 83 empresas brasileiras, no período de 2005 a 2009, foi identificada uma relação negativa entre maior nível de social disclosure e custo de capital próprio mensurado pelo CAPM. Neste estudo, as informações sociais foram avaliadas na perspectiva da avaliação dos programas sociais externos desenvolvidos e/ou apoiados pelas organizações.

Apesar dos resultados dos estudos ora mencionados, verifica-se que em tais pesquisas não se buscaram identificar se o momento da divulgação é relevante quanto à incorporação da informação de natureza social no preço do ativo, o que pode ser realizado por intermédio do estudo de evento.

\subsection{Evento informação e sua repercussão no mercado financeiro}

Para Lamounier e Nogueira (2005) o estudo de eventos tem por objetivo mensurar a repercussão de uma determinada informação, assim que ocorre a sua divulgação, no preço dos ativos/ações. Quando se trata de pesquisas que buscaram avaliar a repercussão de um evento relacionado às ações de responsabilidade social ou que busquem melhorar a reputação corporativa por intermédio de tais ações, as contribuições ainda são incipientes. Por exemplo, os estudos de Dias (2007), Silva, Coelho e Luz (2008) e Caparelli (2010), buscaram explicar os efeitos da adesão do índice de sustentabilidade empresarial da BMF\&Bovespa no retorno anormal das empresas.

Nos achados de Dias (2007), as empresas participantes da carteira ISE, quando comparadas com o grupo de controle, não apresentaram retornos anormais estatisticamente significantes em nenhuma das janelas parametrizadas para o estudo de evento. No estudo de Silva, Coelho e Luz (2008), utiliza-se uma análise temporal no período de 2005 a 2007, sendo constatado que as empresas listadas no ISE não tiveram valorização em razão da referida adesão. Por outro lado, também não sofreram uma desvalorização em razão desse comportamento Já na pesquisa de Caparelli (2010), que utilizou janelas de evento de 15 dias antes do anúncio e 120 dias após o anúncio à Carteira ISE, no ano de 2006, os resultados demonstram que essas empresas obtêm retornos anormais acumulados e estatisticamente positivos e significativos, quando comparadas ao grupo de controle.

Como observado, as pesquisas mencionadas tiveram como preocupação central identificar se o evento - adesão à carteira ISE - repercutiu, positivamente ou não, no preço das ações analisadas. Entretanto, não foram identificados estudos que buscaram avaliar se no evento da divulgação da informação social, houve repercussão na precificação das ações. Assim sendo, esta pesquisa avança em relação às anteriores na medida em que, por meio do estudo de eventos, busca investigar se o evento de divulgação da informação social é determinante para o aumento do retorno acionário em empresas brasileiras abertas.

\section{PROCEDIMENTOS METODOLÓGICOS}

\subsection{Dados e amostra}

Para alcançar o objetivo da pesquisa, foram inicialmente identificadas para a amostra as empresas brasileiras abertas que possuíam atividades de responsabilidade social e que fizeram a sua divulgação por meio dos relatórios anuais e/ou de sustentabilidade. Ainda nesse processo amostral, foram identificadas empresas que no período de 2005 a 2012, tiveram mudanças significativas em sua estrutura patrimonial, provocadas por fusões e incorporações. Se tais fatos ocorreram, as empresas correspondentes foram excluídas da amostra, de modo a manter a sua consistência. Adicionalmente, foram identificadas as empresas com maior liquidez, considerando o 
indicador "liquidez em bolsa", disponibilizado pelo sistema Economática ${ }^{\circledR}$ segundo a equação:

$$
\text { Liquidez em bolsa }=\frac{100 p}{P \sqrt{\frac{n / N}{v / V}}}
$$

em que:

$p$ : número de dias em que houve pelo menos um negócio com a ação dentro do período escolhido;

$P$ : número total de dias do período escolhido;

$n$ : número de negócios com a ação dentro do período escolhido;

$N$ : número de negócios com todas as ações dentro do período escolhido;

$v$ : volume em dinheiro com a ação dentro do período escolhido;

$V$ : volume em dinheiro com todas as ações dentro do período escolhido.

Nesse cálculo, foram incluídas somente as empresas que obtiveram um índice de liquidez em bolsa maior que 0,001 para cada período analisado. Essa restrição decorre do fato de que a ausência de negociação de determinada ação poderia provocar viés na estimação da variável dependente. Posteriormente, buscou-se identificar o momento em que o relatório anual ou de sustentabilidade foram divulgados, a fim de se determinar a data do evento, pois, sem este, não haveria condições de se estabelecer a janela de análise. A data de divulgação foi coletada nas seguintes fontes: a) no calendário de eventos corporativos, b) no próprio relatório em estudo divulgado pela empresa, ou c) por solicitação, via e-mail. As empresas em que não foi possível identificar a data foram excluídas da amostra.

Após a realização desses procedimentos, obteve-se uma amostra com 49 empresas e 254 observações, com a obtenção de uma série temporal não balanceada, haja vista que há empresas que participam da análise, porém, em alguns anos do período de 2005 a 2012 não dispunham de dados para fazer parte em todos os anos. Essa situação ocorre em razão de o evento analisado não possuir uma data específica para sua divulgação, por se tratar de relatórios voluntários, e por isso, não foi possível identificar para todas as empresas a data de divulgação do relatório anual e/ou de sustentabilidade (apesar de terem sido percorridas as etapas de "a" a "c").

\subsection{Variáveis e sua operacionalização}

O nível de social disclosure, por sua vez, foi mensurado por um índice que avalia as informações sociais relacionadas aos programas sociais externos a partir de 13 indicadores, fundamentado em Ramanathan (1976), Haydel (1989) e Hammond e Miles (2004) e validado por Gonçalves (2011). Desse modo, o nível de social disclosure varia em uma escala de pontuação que vai de 13 (todos os indicadores restritos) a 52 pontos (todos os indicadores amplos), conforme o Quadro 1 a seguir.

\begin{tabular}{c|c|l}
\hline & \multicolumn{1}{|c}{ Indice de Social Disclosure } \\
\hline Dimensão & Direcionadores & \multicolumn{1}{c}{ Itens Evidenciados } \\
\cline { 2 - 3 } & Avaliação & VAR001: relação dos programas sociais com os valores da empresa \\
\cline { 2 - 3 } & Avaliação & VAR002: avaliação das necessidades locais para realização dos programas sociais \\
\cline { 2 - 3 } $\begin{array}{c}\text { Informações } \\
\text { passadas }\end{array}$ & Mensuração & VAR005: descrição de objetivos e metas para os programas sociais \\
\cline { 2 - 3 } & Mensuração & $\begin{array}{l}\text { VAR007: valor econômico e/ou financeiro dos recursos humanos envolvidos em } \\
\text { programas de voluntariado }\end{array}$ \\
\cline { 2 - 3 } & Mensuração & $\begin{array}{l}\text { VAR008: valor econômico e/ou financeiro dos recursos materiais destinados aos } \\
\text { programas sociais }\end{array}$ \\
\cline { 2 - 3 } $\begin{array}{c}\text { Prospecções } \\
\text { de ações } \\
\text { futuras }\end{array}$ & Pensuração & VAR009: resultados sociais obtidos nos programas sociais \\
\cline { 2 - 3 } & Avaliação & $\begin{array}{l}\text { VAR003: descrição de aspectos para melhoria dos resultados sociais obtidos nos } \\
\text { programas sociais }\end{array}$ \\
\cline { 2 - 3 } & Mensuração & $\begin{array}{l}\text { VAR010: projeção do fluxo de caixa futuro da alocação de recursos em programas } \\
\text { sociais }\end{array}$ \\
\hline \multirow{3}{*}{\begin{tabular}{c} 
Acessibilidade \\
\cline { 2 - 3 }
\end{tabular}} & Padrão de relatórios & VAR012: disponibilidade dos relatórios de responsabilidade social \\
\cline { 2 - 3 } & Padrão de relatórios & VAR013: frequência da divulgação dos relatórios de responsabilidade social \\
\hline
\end{tabular}

Quadro 1. Indicadores do índice de social disclosure

Fonte: Gonçalves (2011) e (Ramanathan, 1976) 
No que tange a variável - retorno anormal, sua operacionalização está demonstrada no item 3.3, momento no qual também são definidos os parâmetros para o estudo de evento.

\subsection{Estudo de evento}

MacKinlay (1997, p. 14) enumera dois passos principais para se conduzir um estudo de eventos: 1) descrição do procedimento objeto do estudo de evento; 2) definição do período no qual o evento será estudado, ou seja, a janela de eventos. Seguindo os passos de MacKinlay (1997), o presente estudo de evento tem como objeto a análise da reação do mercado diante do ato de divulgação de informações sociais e voluntárias pelas empresas brasileiras, ou seja, análise do retorno anormal das ações quando da realização do social disclosure.

O evento ora analisado é a data de divulgação da informação social, data que varia de empresa para empresa, em razão da não obrigatoriedade desse relatório. Após a identificação das datas em que tal informação foi divulgada, foram definidas as janelas de evento (maior detalhamento sobre a data de divulgação e das janelas de evento podem ser visualizados no Apêndice 1). A definição da janela de evento, envolve certa discricionariedade do pesquisador, podendo variar a partir dos objetivos da pesquisa. Para este estudo, a janela de eventos foi estabelecida a partir de 15 dias de negociação da ação antes do evento, isto é, da data da divulgação da informação social, e de 15 dias de negociação da ação após o evento, para os 8 anos estudados. Para a análise, estas janelas foram dividas em três, conforme discriminado a seguir:

\begin{tabular}{c|c}
\hline Janela & Período da Janela para Análise \\
\hline Janela 1 & -15 a 0 \\
\hline Janela 2 & $0 \mathrm{a}+15$ \\
\hline Janela 3 & $-15 \mathrm{a}+15$ \\
\hline
\end{tabular}

Quadro 2. Período de Evento e Janelas de Eventos

O objetivo da divisão em três janelas, é o de identificar o comportamento do retorno anormal no período que antecede a divulgação do social disclosure (Janela 1), no período imediatamente posterior à divulgação da informação (Janela 2), e análise do comportamento em todo o período, anterior e posterior à divulgação (Janela 3). Para todas as datas compreendidas na janela de eventos, foram calculados os retornos anormais, conforme a equação (2):

$$
A R_{i t}=R_{i t}-R_{m t}
$$

Onde

$$
\begin{aligned}
& A R_{i t} \text { é o retorno anormal da i-ésima ação no período t; } \\
& R_{m t} \text { é o retorno da carteira de mercado (Ibovespa) no período t. }
\end{aligned}
$$

O retorno anormal, caracterizado pelo excesso de retorno de uma determinada ação quando comparada a uma carteira hipotética de mercado, é especialmente útil na medida em que pode capturar o comportamento da ação a partir da percepção do mercado em relação à empresa ora avaliada. No que tange à carteira hipotética de mercado, este estudo utilizou como proxy o retorno médio do Ibovespa, índice também adotado por Medeiros e Silveira (2007), Dias (2007), Silva, Coelho e Luz (2008) e Caparelli (2010).

Ainda no que tange ao cálculo do retorno normal, Fama et. al. (1969) utilizam a seguinte função logarítmica, que torna a distribuição dos retornos simétrica (SOARES; ROSTAGNO; SOARES, 2002):

Onde:

$$
R_{i t}=\ln \left(P_{t} / P_{t-1}\right)
$$

$$
\begin{aligned}
& R_{i t}=\text { retorno normal ou esperado } \\
& P_{t}=\text { cotação da ação em uma data base; } \\
& P_{t-1}=\text { cotação da ação no mercado em um dia anterior; } \\
& \ln (.)=\text { operador de logaritmo natural. }
\end{aligned}
$$

A fim de realizar a análise e teste dos resultados abrangidos pelas janelas de 1 a 3 , foi calculado o retorno anormal acumulado, como propõe Caparelli (2010), conforme a seguir: 


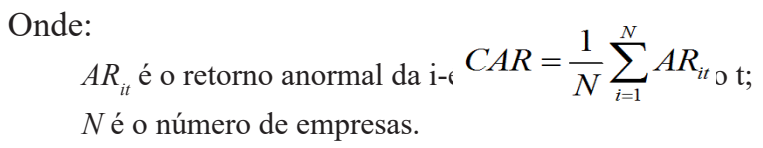

\subsection{Técnicas de análise}

Para a concretização do estudo de eventos, foi realizada inicialmente uma análise descritiva, segregando as empresas por nível de evidenciação, mensurado pelo índice de social disclosure (ISD), e pelo resultado obtido pelo retorno anormal em cada uma das janelas ora mencionadas. O objetivo inicial dessa análise é a de verificar como ficaram distribuídas as empresas, considerando os três fatores de forma conjunta: retorno anormal, nível de evidenciação e evento (janela1, janela 2 e janela 3). A seguir, foi realizado um teste paramétrico de médias (ANOVA). Para a análise de variância, o teste assume que a amostra possui uma distribuição normal com média $\mu_{i}$ e variância homogênea, $Y_{i j}^{\sim} N\left(0, \sigma^{2}\right)$. Caso ocorra violação dos pressupostos para o esse teste, é realizado o teste não paramétrico de Kruskal-Wallis.

\subsection{Limitações}

Quanto ao estudo de evento utilizado para a elaboração deste estudo, McWilliams e Siegel (1997, p. 629) citam três problemas metodológicos principais, que são, na verdade, três premissas adotadas sem que haja a devida comprovação de que ocorrem, quais sejam: 1) os mercados são eficientes (através da hipótese da eficiência de mercado); 2) os eventos divulgados não foram antecipados (anunciados ao mercado antes de sua efetiva divulgação); e 3) a inexistência de outros eventos, durante a janela de eventos, o que também poderiam impactar o mercado (aqui é considerado apenas o social disclosure isoladamente).

\section{ANÁLISE DOS RESULTADOS}

A análise dos resultados tem por propósito investigar se o momento de divulgação da informação social é determinante para a melhoria do retorno acionário das empresas brasileiras de capital aberto. Desse modo, a hipótese inicial é a de que as empresas que possuem um maior nível de social disclosure apresentam um retorno anormal superior quando comparadas às empresas que têm menor nível de evidenciação.

A sustentação desse pressuposto é que uma maior divulgação implica em geração de maior liquidez das ações no mercado (HUANG; XU; LIU, 2014), o que resultaria em diminuição do custo de capital, pela redução do risco sistemático (DHALIWAL et. al., 2011; GHOUL et. al., 2011). Além disso, para Jo e Harjoto (2014) e Cahan et. al. (2014), empresas com as características de alocação de recursos em investimentos sociais e maior divulgação passam a ter maior cobertura por parte da imprensa especializada, e, por conseguinte, recebem avaliações positivas, que passam a contribuir para a sua imagem reputacional.

Para Ioannou e Serafeim (2010), essas avaliações relacionam-se ao fato de que as empresas, ao incorporarem tais práticas em sua estratégia, são percebidas de forma diferenciada em razão de que tais ações reduzem as externalidades provocadas pelas atividades organizacionais e, por consequência, o risco sistemático.

Assim sendo, inicialmente são apresentados os resultados segregados por nível de evidenciação (ISD), pelo retorno anormal considerando a janela $1\left(\mathrm{t}_{-15}\right.$ a $\left.\mathrm{t}_{0}\right)$, a janela $2\left(\mathrm{t}_{0}\right.$ a $\left.\mathrm{t}_{+15}\right)$ e a janela $3\left(\mathrm{t}_{-15}\right.$ a $\left.\mathrm{t}_{+15}\right)$ para o período em análise, de 2005 a 2012. Ressalte-se que, o momento de divulgação é diferente para cada empresa (as datas de divulgação variam para cada uma), o que diminui o risco da ocorrência de que o retorno anormal médio da amostra tenha sido gerado em decorrência de algum fator externo à empresa.

Tabela 1. Retornos anormais no período de 2005 a 2012 por nível de evidenciação 


\begin{tabular}{|c|c|c|c|c|c|c|c|}
\hline \multirow[b]{2}{*}{ Janela } & \multicolumn{3}{|c|}{ Retorno Anormal 2005} & \multirow[b]{2}{*}{ Janela } & \multicolumn{3}{|c|}{ Retorno Anormal 2006} \\
\hline & $\begin{array}{c}1^{\circ} \text { Tercil } \\
\text { ISD } 20\end{array}$ & $\begin{array}{c}2^{\circ} \text { Tercil } \\
\text { ISD } 24 \\
\end{array}$ & $\begin{array}{c}3^{\circ} \text { Tercil } \\
\text { ISD } 30\end{array}$ & & $\begin{array}{c}1^{\circ} \text { Tercil } \\
\text { ISD 22,75 }\end{array}$ & $\begin{array}{c}2^{\circ} \text { Tercil } \\
\text { ISD 26,50 }\end{array}$ & $\begin{array}{c}3^{\circ} \text { Tercil } \\
\text { ISD } 32\end{array}$ \\
\hline $\mathbf{t}_{-15} \mathbf{a t}_{0}$ & $-0,003139$ & 0,000542 & 0,004078 & $\mathbf{t}_{-15} \mathbf{a t}_{\mathbf{0}}$ & $-0,003577$ & 0,000688 & 0,002796 \\
\hline $\mathbf{t}_{0} \mathbf{a} \mathbf{t}_{+15}$ & $-0,0025989$ & $-0,00010$ & 0,006079 & $\mathbf{t}_{\mathbf{0}} \mathbf{a} \mathbf{t}_{+15}$ & $-0,000977$ & 0,009779 & 0,003817 \\
\hline $\mathbf{t}_{-15} \mathbf{a} \mathbf{t}_{+15}$ & 0,0018287 & 0,000989 & 0,002714 & $\mathbf{t}_{-15}$ a $\mathbf{t}_{+15}$ & $-0,001862$ & $-0,000170$ & 0,003778 \\
\hline \multirow[b]{2}{*}{ Janela } & \multicolumn{3}{|c|}{ Retorno Anormal 2007} & \multirow[b]{2}{*}{ Janela } & \multicolumn{3}{|c|}{ Retorno Anormal 2008} \\
\hline & $\begin{array}{c}1^{\circ} \text { Tercil } \\
\text { ISD 22,50 }\end{array}$ & $\begin{array}{l}2^{\circ} \text { Tercil } \\
\text { ISD } 28\end{array}$ & $\begin{array}{l}3^{\circ} \text { Tercil } \\
\text { ISD 33,5 }\end{array}$ & & $\begin{array}{c}1^{\circ} \text { Tercil } \\
\text { ISD } 20\end{array}$ & $\begin{array}{c}2^{\circ} \text { Tercil } \\
\text { ISD } 24\end{array}$ & $\begin{array}{l}3^{\circ} \text { Tercil } \\
\text { ISD 29,5 }\end{array}$ \\
\hline$t_{-15} a t_{0}$ & $-0,002428$ & 0,000219 & 0,004009 & $\mathbf{t}_{-15} \mathbf{a} \mathbf{t}_{\mathbf{0}}$ & $-0,005276$ & $-0,001994$ & 0,000943 \\
\hline $\mathbf{t}_{\mathbf{0}} \mathbf{a} \mathbf{t}_{+15}$ & $-0,003210$ & 0,000272 & 0,000242 & $\mathbf{t}_{\mathbf{0}} \mathbf{a} \mathbf{t}_{+15}$ & $-0,004010$ & $-0,002521$ & 0,003465 \\
\hline $\mathbf{t}_{-15} \mathbf{a} \mathbf{t}_{+15}$ & $-0,002548$ & $-0,000048$ & 0,002617 & $\mathbf{t}_{-15}$ a $\mathbf{t}_{+15}$ & $-0,003653$ & $-0,000865$ & 0,000387 \\
\hline \multirow[b]{2}{*}{ Janela } & \multicolumn{3}{|c|}{ Retorno Anormal 2009} & \multirow[b]{2}{*}{ Janela } & \multicolumn{3}{|c|}{ Retorno Anormal 2010} \\
\hline & $\begin{array}{c}1^{\circ} \text { Tercil } \\
\text { ISD } 24,75 \\
\end{array}$ & $\begin{array}{l}2^{\circ} \text { Tercil } \\
\text { ISD 30,5 }\end{array}$ & $\begin{array}{c}3^{\circ} \text { Tercil } \\
\text { ISD } 35\end{array}$ & & $\begin{array}{l}1^{\circ} \text { Tercil } \\
\text { ISD } 26\end{array}$ & $\begin{array}{c}2^{\circ} \text { Tercil } \\
\text { ISD } 33\end{array}$ & $\begin{array}{c}3^{\circ} \text { Tercil } \\
\text { ISD } 36 \\
\end{array}$ \\
\hline$t_{-15} a t_{0}$ & $-0,003813$ & $-0,001219$ & 0,001732 & $\mathbf{t}_{-15} \mathbf{a t}_{\mathbf{0}}$ & $-0,001430$ & 0,000492 & 0,003235 \\
\hline $\mathbf{t}_{0} \mathbf{a} \mathbf{t}_{+15}$ & $-0,005715$ & $-0,000540$ & 0,002074 & $\mathbf{t}_{\mathbf{0}} \mathbf{a} \mathbf{t}_{+15}$ & $-0,002691$ & 0,000225 & 0,004338 \\
\hline $\mathbf{t}_{-15} \mathbf{a t ~ t}_{+15}$ & $-0,004284$ & $-0,001427$ & 0,001419 & $\mathbf{t}_{-15}$ a $\mathbf{t}_{+15}$ & $-0,002161$ & 0,000328 & 0,002503 \\
\hline \multirow[b]{2}{*}{ Janela } & \multicolumn{3}{|c|}{ Retorno Anormal 2011} & & \multicolumn{3}{|c|}{ Retorno Anormal 2012} \\
\hline & $\begin{array}{c}1^{\circ} \text { Tercil } \\
\text { ISD 25,25 }\end{array}$ & $\begin{array}{c}2^{\circ} \text { Tercil } \\
\text { ISD } 31,50\end{array}$ & $\begin{array}{c}3^{\circ} \text { Tercil } \\
\text { ISD } 37\end{array}$ & Janela & $\begin{array}{c}1^{\circ} \text { Tercil } \\
\text { ISD } 32\end{array}$ & $\begin{array}{c}2^{\circ} \text { Tercil } \\
\text { ISD } 34\end{array}$ & $\begin{array}{c}3^{\circ} \text { Tercil } \\
\text { ISD } 37\end{array}$ \\
\hline $\mathbf{t}_{-15}$ a $\mathbf{t}_{0}$ & $-0,002582$ & 0,000471 & 0,002728 & $\mathbf{t}_{-15}$ a $\mathbf{t}_{0}$ & $-0,001364$ & 0,000425 & 0,002163 \\
\hline $\mathbf{t}_{0} \mathbf{a} \mathbf{t}_{+15}$ & $-0,001788$ & 0,002083 & 0,006321 & $\mathbf{t}_{\mathbf{0}} \mathbf{a} \mathbf{t}_{+15}$ & $-0,003702$ & 0,000547 & 0,004237 \\
\hline $\mathbf{t}_{-15} \mathbf{a} \mathbf{t}_{+15}$ & $-0,000610$ & 0,001157 & 0,004291 & $\mathbf{t}_{-15}$ a $\mathbf{t}_{+15}$ & $-0,002354$ & 0,001439 & 0,002416 \\
\hline \multirow[b]{2}{*}{ Janela } & \multicolumn{3}{|c|}{ Retorno Anormal 2005-2012 } & & & & \\
\hline & $\begin{array}{c}1^{\circ} \text { Tercil } \\
\text { ISD } 23\end{array}$ & $\begin{array}{c}2^{\circ} \text { Tercil } \\
\text { ISD } 29\end{array}$ & $\begin{array}{c}3^{\circ} \text { Tercil } \\
\text { ISD } 35\end{array}$ & & & & \\
\hline$t_{-15} a t_{0}$ & $-0,0029370$ & 0,000079 & 0,002606 & & & & \\
\hline $\mathbf{t}_{0} \mathbf{a t ~ t}_{+15}$ & $-0,0036479$ & 0,000225 & 0,0037151 & & & & \\
\hline $\mathbf{t}_{-15} \mathbf{a} \mathbf{t}_{+15}$ & $-0,002428$ & 0,000003 & 0,0024936 & & & & \\
\hline
\end{tabular}

Primeiramente, destaque-se que os valores dos retornos anormais descritos na tabela anterior encontramse na forma logarítmica. Esses retornos foram divididos em tercis, por nível de evidenciação (ISD). Além disso, para as empresas classificadas nos respectivos tercis, foram analisados os retornos anormais para cada janela, isto é, janela $1\left(\mathrm{t}_{-15} \mathrm{a} \mathrm{t}_{0}\right)$, que antecede a divulgação; janela $2\left(\mathrm{t}_{0} \mathrm{at}_{+15}\right)$, imediatamente posterior à divulgação; e janela $3\left(\mathrm{t}_{-15}\right.$ a $\left.\mathrm{t}_{+15}\right)$, que compreende todo o período.

Pode-se observar, inicialmente, que de um modo geral as empresas com maior nível de social disclosure (ISD) tiveram um retorno anormal maior em todas as janelas. Isto é, há uma tendência de que, quanto maior o índice de disclosure, maior o retorno positivo ou menor o retorno negativo (de forma absoluta), corroborando os estudos de Poddi e Vergali (2009) e Oikonomou, Brooks e Pavelin (2014). Essa característica só não foi observada no ano de 2006 na janela $2\left(\mathrm{t}_{0} \mathrm{a} \mathrm{t}_{+15}\right)$, em que as empresas do segundo tercil tiveram um melhor desempenho quando comparado às empresas do terceiro tercil.

Analisando o retorno anormal acumulado para o período de 2005 a 2012, observa-se que, na janela $1\left(t_{-15}\right.$ a $t_{0}$ ), houve retorno anormal negativo para o primeiro tercil (menor nível de evidenciação), enquanto que para $\mathrm{o}$ $3^{\circ}$ tercil, houve um retorno anormal positivo superior ao $2^{\circ}$ tercil. O retorno anormal apresentou melhora após a divulgação da informação social (janela 2), tanto para o segundo e terceiro tercis, enquanto que apenas o primeiro, que possui o menor nível de evidenciação, apresentou piora. Esse fato vai ao encontro da hipótese de que um maior nível de social disclosure pode levar a um favorecimento por parte do mercado financeiro na precificação dos ativos, podendo tal fato ocorrer pelo que Jo e Harjoto (2014) e Cahan et. al. (2014) afirmam acerca da maior cobertura pelos analistas e melhores avaliações, resultando em uma melhor reputação corporativa.

Os resultados encontrados para cada ano corroboram a tendência de que a divulgação da informação pode incrementar o retorno. Após a divulgação social, ou seja, na janela $2\left(\mathrm{t}_{0}\right.$ a $\left.\mathrm{t}_{+15}\right)$, pode-se observar que em quase todos os tercis houve um aumento do retorno anormal, se comparado à janela anterior à divulgação, ou 
seja, a janela $1\left(\mathrm{t}_{-15}\right.$ a $\left.\mathrm{t}_{0}\right)$, quando são analisados ano a ano.

É interessante observar, porém, que alguns anos apresentaram resultados fora do padrão ora mencionado, como é o caso do ano de 2007. Pois tanto o primeiro tercil quanto o terceiro tiveram uma piora no retorno anormal após a divulgação da informação social, ocorrendo isso também no ano de 2008, para o segundo tercil, no ano de 2009, para o primeiro tercil e no ano 2010, para o primeiro e segundo tercis.

Apesar disso, a tendência observada é a de que o primeiro tercil apresenta piora em seus retornos em quatro anos, o segundo, em dois, e o terceiro, em apenas um, ou seja, de forma geral, quanto maior o nível de social disclosure, menor será o retorno negativo, ou um maior retorno positivo, sobretudo na janela posterior à divulgação (janela 2).

A fim de testar a hipótese de que as empresas abertas que possuem maior nível de social disclosure apresentam retornos anormais médios superiores e estatisticamente significativos, foram realizados testes de normalidade da amostra e homogeneidade das variâncias. Os resultados podem ser observados na tabela a seguir.

Tabela 2. Teste de Normalidade KS e de Homogeneidade das Variâncias de Levene

\begin{tabular}{l|c|c|c|c}
\hline \multicolumn{1}{c|}{ Teste } & ISD & $\begin{array}{c}\text { Janela 1 } \\
\mathbf{t}_{-15} \mathbf{a ~ t}_{\mathbf{0}}\end{array}$ & $\begin{array}{c}\text { Janela 2 } \\
\mathbf{t}_{\mathbf{0}} \mathbf{a ~ t}_{+15}\end{array}$ & $\begin{array}{c}\text { Janela 3 } \\
\mathbf{t}_{-15} \mathbf{a ~ t}_{+15}\end{array}$ \\
\hline $\mathrm{N}$ & 254 & 254 & 254 & 254 \\
\hline Kolmogorov-Smirnov Z & 1,240 &, 660 & 2,514 & 2,199 \\
\hline p-valor & 0,092 & 0,777 & 0,000 & 0,000 \\
\hline Levene Statistic & & 2,309 & 1,933 & 1,721 \\
\hline p-valor & & 0,000 & 0,004 & 0,015 \\
\hline
\end{tabular}

Os resultados do teste $\mathrm{KS}$ evidenciam normalidade para a realização do teste de variância para o índice de social disclosure (ISD) na janela 1, e uma distribuição não normal para as janelas 2 e 3 . Ocorre que o teste de anova parte do pressuposto da homogeneidade das variâncias, o que não ocorreu em todas as janelas (1, 2 e 3), conforme pode ser observado pelo teste de Levene ( $\mathrm{p}$-valor $<0,05$ ).

Em razão dos resultados obtidos, foi realizado o teste não paramétrico de Kruskal-Wallis (KW), cuja $\mathrm{H}_{0}$ é de que as distribuições amostrais são iguais.

Tabela 3. Teste de Média de Kruskal-Wallis para nível de social disclosure e retorno anormal

\begin{tabular}{l|c|l|c|l|c}
\hline Período & \multicolumn{5}{|c}{ 2005-2012 } \\
\hline Janela & 1 & Janela & 2 & Janela & 3 \\
\hline Período & $\mathrm{t}_{-15} \mathrm{at}_{0}$ & Período & $\mathrm{t}_{0} \mathrm{a}_{+15}$ & Período & $\mathrm{t}_{-15} \mathrm{a} \mathrm{t}_{+15}$ \\
\hline Qui-quadrado & 41,172 & Qui-quadrado & 31,949 & Qui-quadrado & 34,854 \\
\hline p-valor & 0,084 & p-valor & 0,370 & p-valor & 0,248 \\
\hline
\end{tabular}

Os resultados do teste KW apontam uma não rejeição da hipótese nula, isto é, apesar de as empresas da amostra que possuem maior nível de social disclosure apresentarem um retorno anormal médio superior quando comparado às empresas com menor nível de evidenciação, esse aspecto não é estatisticamente significante no período (2005 a 2012), nas respectivas janelas de evento analisadas.

Como foram identificados retornos anormais superiores para as empresas que obtiveram um maior nível de social disclosure, tal como os achados de Caparelli (2010), que identificou um retorno anormal significativo em janelas próximas ao evento analisado $\left(t_{0}\right.$ a $t_{+1}$ e $t_{-2}$ a $\left.t_{+2}\right)$, foram definidas duas janelas adicionais - janelas $4\left(t_{-1}\right.$ $\left.\mathrm{at}_{+1}\right)$ e $5\left(\mathrm{t}_{-2} \mathrm{at}_{+2}\right)$.

Além disso, considerando o pressuposto do estudo de que maior divulgação pode implicar em maior liquidez das ações no mercado (HUANG; XU; LIU, 2014), foram analisados os resultados somente para os anos em que as empresas obtiveram maior nível de social disclosure como um todo, quais sejam: 2006, 2007, 2009 , 2010 e 2011. A seguir, é apresentada a análise descritiva dos dados, sendo os retornos anormais segregados por tercis entre as janelas de 1 a 5, bem como as variáveis - social disclosure e liquidez.

Tabela 4. Descrição por tercis das variáveis liquidez, social disclosure e retornos anormais 


\begin{tabular}{c|c|c|c|c|c|c|c}
\hline $\begin{array}{c}\text { Percentis } \\
\mathrm{n}=168\end{array}$ & $\begin{array}{c}\text { Liquidez } \\
\text { do Ativo }\end{array}$ & $\begin{array}{c}\text { Social } \\
\text { Disclosure }\end{array}$ & $\begin{array}{c}\text { Janela 1 } \\
\mathrm{t}_{-15} \mathrm{a} \mathrm{t}_{0}\end{array}$ & $\begin{array}{c}\text { Janela } 1 \\
\mathrm{t}_{0} \mathrm{at}_{+15}\end{array}$ & $\begin{array}{c}\text { Janela 3 } \\
\mathrm{t}_{-15} \mathrm{t}_{+15}\end{array}$ & $\begin{array}{c}\text { Janela 4 } \\
\mathrm{t}_{-1} \mathrm{at}_{+1}\end{array}$ & $\begin{array}{c}\text { Janela } 5 \\
\mathrm{t}_{-2} \mathrm{at}_{+2}\end{array}$ \\
\hline 25 & 0,026 & 24 & $-0,002844$ & $-0,00349$ & $-0,00244$ & $-0,01091$ & $-0,00630$ \\
\hline 50 & 0,154 & 29,50 & 0,000257 & 0,00033 & 0,00022 & $-0,00149$ & $-0,00050$ \\
\hline 75 & 0,518 & 35,00 & 0,002522 & 0,00356 & 0,00271 & 0,00691 & 0,00586 \\
\hline
\end{tabular}

A partir da Tabela 4, verifica-se que, em todas as janelas as empresas que tiveram maior nível de social disclosure obtiveram um melhor retorno anormal e maior liquidez de seus ativos, corroborando à hipótese inicial de que empresas com maior nível de social disclosure têm um maior retorno anormal quando comparadas as empresas que possuem um menor nível de evidenciação (HUANG; XU; LIU, 2014). A seguir, é realizado o teste de hipótese, com o objetivo de revelar se as referidas diferenças são estatisticamente significantes.

Tabela 5. Teste de Média de Kruskal-Wallis para nível de social disclosure e retorno anormal

\begin{tabular}{l|c|c|l|c|c}
\hline Período & \multicolumn{2}{|c|}{ 2005-2012 } & \multicolumn{2}{c}{ 2006-2007-2009-2010-2011 } \\
\hline Janela & 4 & 5 & Janela & 4 & 5 \\
\hline Período & $\mathrm{t}_{-1} \mathrm{a}_{+1}$ & $\mathrm{t}_{-2} \mathrm{a}_{+2}$ & Período & $\mathrm{t}_{-1} \mathrm{a}_{+1}$ & $\mathrm{t}_{-2} \mathrm{a}_{+2}$ \\
\hline Qui-quadrado & 41,559 & 41,367 & Qui-quadrado & 40,747 & 43,689 \\
\hline p-valor & 0,078 & 0,081 & p-valor & 0,072 & 0,039 \\
\hline
\end{tabular}

Corroborando os achados de Caparelli (2010), ao nível de 5\% de significância, $\mathrm{H}_{0}$ é rejeitada na janela $5\left(\mathrm{t}_{-2}\right.$ a $\left.\mathrm{t}_{+2}\right)$ para o período em que foi apresentado um maior desempenho das empresas no que tange ao nível de evidenciação. Ainda nas janelas $5\left(\mathrm{t}_{-2}\right.$ a $\left.\mathrm{t}_{+2}\right)$, de ambos os períodos, e na janela 4, para o período de 2005-2012, $\mathrm{H}_{0}$ é rejeitada ao nível de significância de $10 \%$.

Esses resultados apontam para pelo menos dois aspectos importantes acerca do comportamento do mercado acionário: a) que há uma reação em relação às informações disponíveis em momentos muito próximos ao evento; e b) que a informação acerca dos programas sociais externos avaliados neste estudo pelo índice de social disclosure é relevante e rapidamente incorporada ao preço do ativo.

Os resultados vão ao encontro do que Richardson, Welker e Hutchinson (1999), Orlitzky e Benjamin (2001), Brown, Helland e Smith (2006) afirmam a respeito da contribuição das ações estratégicas de responsabilidade social para a melhoria da imagem organizacional, a ponto de maximizar o valor das ações, diminuir o risco e trazer vantagens competitivas.

Adicionalmente, verifica-se que tais ações possuem a condição de contribuir com a maximização do retorno das empresas ao longo do tempo, considerando o período analisado e o comportamento identificado em todas as janelas de evento, corroborando com o que Saiia, Carroll e Buchholtz (2003), Jo e Harjoto (2014) e Cahan et. al. (2014) afirmam sobre a capacidade que essas ações têm, ao serem incorporadas como estratégias organizacionais, de servirem como instrumento de melhoria da imagem organizacional e de redução do risco sistemático.

Além disso, muito embora não haja um momento pré-determinado para que a referida informação seja disponibilizada, verifica-se que o mercado acionário acompanha as ações empresariais não somente quando da divulgação da informação, mas também em períodos próximos à ocorrência do evento.

Vale destacar que os resultados aqui encontrados acerca da janela de evento tornam-se mais relevantes quando observados sob a ótica de que ocorreram em momentos distintos para cada uma das empresas, e, sobretudo, que foram testados ao longo de um período de 8 anos (2005 a 2012).

\section{CONSIDERAÇÕES FINAIS}

Com o objetivo de investigar se o momento da divulgação da informação social (SD) é determinante para a melhoria do retorno acionário das empresas brasileiras de capital aberto, foram analisadas 49 empresas abertas e 254 observações no período de 2005 a 2012.

O desenvolvimento metodológico compreendeu a análise de conteúdo dos relatórios anuais e/ou de 
sustentabilidade, de modo a avaliá-los e mensurá-los, para a obtenção do nível de social disclosure. Além disso, foram identificadas as datas de divulgação dos referidos relatórios, que, por serem de natureza voluntária, não possuem datas pré-definidas ou uniformes, variando desse modo de empresa para empresa (vide Apêndice 1).

Por meio da análise descritiva, observou-se que as empresas com maior nível de social disclosure obtiveram, em suas ações, maior liquidez e retorno anormal médio acumulado.

Os resultados do teste KW apontaram a não rejeição da hipótese nula, isto é, apesar dos resultados observados na análise descritiva, esse aspecto não é estatisticamente significante, no período de 2005 a 2012, nas respectivas janelas de evento $-1\left(\mathrm{t}_{-15}\right.$ a $\left.\mathrm{t}_{0}\right) ; 2\left(\mathrm{t}_{0}\right.$ a $\left.\mathrm{t}_{+15}\right)$ e $3\left(\mathrm{t}_{-15} \mathrm{at}_{+15}\right)$.

Adicionalmente, foram testadas duas novas janelas $-4\left(\mathrm{t}_{-1}\right.$ a $\left.\mathrm{t}_{+1}\right)$ e $5\left(\mathrm{t}_{-2} \mathrm{at}_{+2}\right)-$ sob as hipóteses de que o mercado financeiro reage rapidamente ao evento analisado quando há um maior nível de evidenciação. Há indícios, também, de que empresas com maiores níveis de social disclosure possuem maior liquidez em suas ações.

Ao nível de $5 \%$ de significância, $\mathrm{H}_{0}$ foi rejeitada na janela $5\left(\mathrm{t}_{-2}\right.$ a $\left.\mathrm{t}_{+2}\right)$ para o período em que as empresas apresentaram maior nível de evidenciação (2006-2007-2009-2010-2011). Tal resultado demonstra que o mercado financeiro reage à maiores níveis de evidenciação e incorpora-os rapidamente ao preço do ativo. Ainda na Janela $5\left(\mathrm{t}_{-1} \mathrm{a}_{+1}\right), \mathrm{H}_{0}$ também foi rejeitada ao nível de significância de $10 \%$, para o mesmo período.

A partir do comportamento observado em todos os anos analisados (2005 a 2012), constata-se que, à medida que as empresas incorporam estratégias de responsabilidade social e realizam sua divulgação, esta contribui para uma melhoria do retorno, se comparado às empresas que possuem em média um menor nível de divulgação. Esse resultado justifica-se, dentre outros fatores, por tais empresas serem observadas com um maior capital reputacional em suas ações gerenciais.

Os resultados desta pesquisa contribuem para compreender o comportamento do mercado financeiro diante da divulgação da informação social, e avança em relação aos estudos anteriores, ao valer-se do método de estudo de eventos para a realização de sua análise. Destaca-se que as conclusões ora extraídas estão restritas à amostra ora analisada, bem como as limitações quanto ao método de estudo de evento e o uso da técnica de análise de conteúdo na avaliação das informações avaliadas (social disclosure).

\section{REFERÊNCIAS}

ANDERSON, J. C.; FRANKLE, A. W. Voluntary social reporting: an iso-beta portfolio analysis. The Accounting Review, Sarasota, v. 55, n. 33, p. 467-479, Jul., 1980.

AHMED, S. U. et al. Institutional investment and corporate social performance: linkage towards sustainable development. Corporate Social Responsibility and Environmental Management, v. 21, n. 1, p. 1-13, 2014.

ARCAY, R. B.; VÁZQUEZ, F. M. Corporate characteristics, governance rules and the extent of voluntary disclosure in Spain. Advance in Accounting, vol. 21, pp. 299-331, 2005.

BALL, R.; BROWN, P.. An Empirical Evaluation of Accounting Income Numbers. Journal of Accounting Research, v. 6, 1968, p. 159-177.

BARDIN, L.. Análise de Conteúdo. Lisboa: Edições 70, 1977.

BRIGHAM, E. E.; GAPENSKI, L. C.; EHRHARD, M. C. Administração financeira: teoria e prática. São Paulo: Atlas, 2001.

BROWN, W. O.; HELLAND, E.; SMITH, J. K.. Corporate Philanthropic Pratices. Journal of Corporate Finance, v. 12,2006, p.855-877.

BRANCO, Manuel Castelo; RODRIGUES, Lúcia Lima. Factors Influenting Social Responsibility Disclosure by Portuguese Companies. Journalof Business Ethics, vol. 83, 2008, p. 685-701.

CAHAN, S. F. et al. The Business Press and Corporate Social Responsibility: Reputation, Cost of Capital, and Firm Value. Working Paper. Disponível em <https://www.business.uq.edu.au/sites/default/files/event/ supportingDocs/chen-chen-paper.pdf $>$, em 11 abr. 2014.

CAMARGOS, M. A.; BARBOSA, F. V.. Fusões e aquisições de empresas brasileiras: criação de valor e sinergias operacionais. RAE - Revista de Administração de Empresas, v. 49, n. 2, abr./jun. 2009, p. 206-220.

CAPARELLI, C. E. Sustentabilidade e retorno ao acionista: um evento sobre o índice de sustentabilidade 
empresarial. XIII SEMEAD - Seminários em Administração. Set. 2010.

CARR, I.; OUTHWAITE, O.. Investigatins the Impact of Anti-Corruption Strategies on International Business: An Interim Report. Disponível em SSRN: <http://ssrn.com/abstract=1410642>. Acesso em: 12 de Julho de 2013, 2009.

CHENG, B.; IOANNOU, I.; SERAFEIM, G.. Corporate social responsibility and access to finance. Strategic Management Journal, v. 35, n. 1, p. 1-23, 2014.

CRISÓSTOMO, V. L. FREIRE, F. S. VASCONCELLOS, F. C. Corporate social responsibility, firmvalueand financial performance in Brazil. Social Responsibility Journal, vol. 7, n. 2, pp. 295-309. 2011.

DE MEDEIROS, O.; SILVEIRA, M. L.. Corporate Values and Stock Performance of Latin American Banks. 2007. Disponível em SSRN: <http://ssrn.com/abstract=958897>. Acesso em: 12 de Julho de 2013.

DEEGAN, C.. The legitimising effect of social and environmental disclosures - a theoretical foundation. Accounting, Auditing\&AccountabilityJournal, vol. 15, pp. 282-311, 2002.

DIAS, E. A. Índice de Sustentabilidade Empresarial e retorno ao acionista: Um estudo de evento. 2007. Dissertação de Mestrado em Administração de Empresas, Universidade Presbiteriana Mackenzie, São Paulo, p. 137, 2007.

DHALIWAL, D. et. al.. Voluntary Non-Financial Disclosure and the Cos of Equity Capital: The Initiation of Corporate Social Responsibility Reporting. The Accounting Review, (January), Vol. 86, N.1, 2011, p.59-100.

EPSTEIN, M. J.; FREEDMAN, M.. Social Disclosure and the Individual Investor. Accounting, Auditing and Accountability Journal, vol. 7 N. (4), pp. 94-109, 1994.

FAMA, E. F. Efficient Capital Markets: A Review of Theory and Empirical Work. The Journal of Finance, vol. 25 , n. 2, May, 1970, p. 383-417.

FRIEDMAN, M. The social responsibility of business is increase its profits. The New York Times Magazine, vol. 13, Sep., 1970.

GANA, M. R., DAKHLAOUI, M. Societal Information Disclosure and The Cost of Equity: The Case of Tunisian Companies. Global JournalsInc, Vol. 11, N. 7, jul, 2011.

GOLlIER, C.; POUGET, S.. The "Washing Machine": Investment Strategies and Corporate Behavior with Socially Responsible Investors. Institut d'Économie Industrielle (IDEI), Toulouse, 2014.

GONÇALVES, R. de S.. Social disclosure e custo de capital próprio em empresas brasileiras. 165. Tese (doutorado) - Faculdade de Economia, Administração e Ciências Contábeis, Universidade de Brasília, Brasília.

GONÇALVES, R. de S. et. al. Social Disclosure e Custo de Capital Próprio em Companhias Abertas no Brasil. R. Cont. Fin. - USP, v. 24, n. 62, maio/jun./jul./ago., 2013, p. 113-124.

HAMMOND, K.; MILES, S.. Assessing quality assessment of corporate social reporting: UK perspectives. AccountingForum, vol. 28, p. 61-79, 2004.

HAYDEL, B. F. A Administração Estratégica de Programas de Responsabilidade Social em Empresas Multinacionais: Percepções da Alta Diretoria. Revista de Administração de Empresas, n. 29, vol. 3, jul./set., p. 5-291989..

HERREMANS, I. M.; AKATHAPORN, P., McINNES, M.. An investigation of corporate social responsibility reputation and economic performance. Accounting Organization and Society, vol. 18, n. 7/8, p. 587-604, 1993.

HILLMAN, A. J.; KEIM, G. D. Shareholder value, stakeholder management, and social issues: what's bottom line? Strategic Management Journal, vol. 22, p. 125-139, 2001.

HUANG, J.; XU, S.; LIU, D.. Empirical Study on the Effects of Corporate Social Responsibility Disclosure in Stock Liquidity. Advances in information Sciences and Service Sciences, vol. 6, n. 1, Feb, p. 62-69, 2014.

IOANNOU, I.; SERAFEIM, G.. THE IMPACTOF CORPORATE SOCIALRESPONSIBILITY ON INVESTMENT RECOMMENDATIONS. In: Academy of Management Proceedings. Academy of Management, 2010, p. 1-6.

JENSEN, M. C. Value maximization, stakeholder theory, and the corporate objective function. JournalofApplied Corporate Finance, Fall, 2001. 
JO, H.; HARJOTO, M.. Analyst coverage, corporate social responsibility, and firm risk. Business Ethics: A European Review, v. 23, n. 3, p. 272-292, 2014.

YEN, Meng-Feng et al. Does Corporate Social Responsibility Deliver Alpha?.Journal of Economics and Management, v. 11, n. 1, p. 23-45, 2015.

LAMOUNIER, W.M.; NOGUEIRA, E. M. Estudo de Eventos: Procedimentos e Estudos Empíricos. In: II Seminário de Gestão de Negócios, 2005, Curitiba. II Seminário de Gestão de Negócios - Um Enfoque Acadêmico da Realidade Empresarial, 2005.

LOURENÇO, Isabel Costa et al. The value relevance of reputation for sustainability leadership. Journal of Business Ethics, v. 119, n. 1, p. 17-28, 2014.

MACHADO FILHO, C. A. P.; ZYLBERSZTAJN, D.. Responsabilidade Social Corporativa e a Criação de Valor para as Organizações. Faculdade de Economia, Administração e Contabilidade da Universidade de São Paulo, Série de WorkingPapers, WorkingPaper n.03/024. 2003. Disponível em $<$ http://www.ead.fea.usp.br/ wpapers> Acesso em 31 mar. 2010.

MacKINLAY, A. C. Event Study in Economics and Finance. Journal of Economic Literature, vol. 35, n. 1, p. 1339, Mar. 1997.

MACKEY, Alison; MACKEY, Tyson B.; BARNEY, Jay B. Corporate social responsibility and firm performance: Investor preferences and corporate strategies. Academy of management review, v. 32, n. 3, p. 817-835, 2007.

McWILliAMS, A.; SIEGEL, D.. Event Studies in Management Research: Theoretical and Empirical Issues. Academy of Management Journal, v. 40, n. 3, p. 98-107, 1997.

McWILLIAMS, A.; SIEGEL, D.; TEOH, S. H.. Issues in the use of the event study methodology: a critical analysis of corporate social responsibility studies. Organizational Research Methods, vol. 2, n. 4, p.340-365, October 1999.

NG, A. C.; REZAEE, Z. Sustainability Disclosures and Cost of Capital. 2012. Disponível em SSRN < http://ssrn. com/abstract=2038654> Acesso em 12 mar. 2013.

ORLITZKY, M.; BENJAMIN, J. D. Corporate Social Performance and Firm Risk: A Meta-Analytic Review. Business Society, vol. 40, n.4, p.369-396, 2001.

OIKONOMOU, I.; BROOKS, C.; PAVELIN, S. The effects of corporate social performance on the cost of corporate debt and credit ratings. Financial Review, v. 49, n. 1, p. 49-75, 2014.

PATTEN, Dennis M. Exposure, Legitimacy, and Social Disclosure. Journal of Accounting and Public Policy, v. 10, p. 297-308, 1991.

PODDI, L.; VERGALLI, S. Does Corporate Social Responsibility Affect the Performance of Firms? Nota Di Lavoro 52.2009. Disponível em <http://ssrn.com/abstract=1444333> Acesso em 14 abr. 2009.

PORTER; M. E.; KRAEMER, M. R. The Competitive Advantage of Corporate Philanthropy. Harvard Business Review, v. 80, n. 12, p.56-68, 2002.

RAMANATHAN, K. V. Toward a theory of corporate social accounting. The Accounting Review, Sarasota, v. 51, n. 3, Jul., p. 516-528, 1976.

REVERTE, C.. The Impact of Better Corporate Social Responsibility Disclosure on the Cost of Equity Capital. Corp. Soc. Responsib. Environ. Mgmt., v. 19, jun., p.253-272, 2012.

RICHARDSON, Alan J.; WELKER, Michael; HUTCHINSON, Ian R. Managing capital market reactions to corporate social responsibility. International Journal of Management Reviews, v. 1, p.17-43, 1999.

ROVER, Suliani; MURCIA, Fernando Dal-Ri. Influência do Disclosure Voluntário Econômico e Socioambiental no Custo de Capital Próprio das Empresas Brasileiras. In. Encontro da Associação Nacional dos Programas de pós graduação em Contabilidade (ANPCONT), 4., 2010, Natal, Anais... Natal: ANPCONT, 2010, 1, CDROM.

ROCKNESS, Joanne; WILLIAMS, Paul F. A descriptive study of social responsibility mutual funds, Accounting, Organizations and Society, vol. 13, Issue 4, p. 397-411, 1988.

SAIIA, David H.; CARROLL, Archie B.; BUCHHOLTZ, Ann K. Philanthropy as Strategy: when Corporate 
Charity “Begins at Home”. Business \&Society, v. 42, n. 2, Jun., p. 169-201, 2003.

SERVAES, H.; TAMAYO, A. The Impact of Corporate Social Responsibility on Firm Value: The Role of Customer Awareness. Management Science, vol. 59, n. 5, May, p. 1045-1061, 2013.

SILVA, L. S. A.; QUELHAS, O. L. G. Sustentabilidade Empresarial e o Impacto no Custo de Capital Próprio das Empresas de Capital Aberto. Gestão \& Produção, v. 13, n. 3, set/dez, p. 385-395, 2006.

SILVA, R.; COELHO, P.; LUZ, S. Impacto da Divulgação do Índice de Sustentabilidade Empresarial sobre os preços das ações: Um Estudo de Eventos nos anos de 2005 a 2007. In. Congresso USP de Controladoria e Contabilidade, São Paulo, 2008.

STERNBERG, E.. The Stakeholder Concept: A Mistaken Doctrine. Foundation for Business Responsibilities, Issue Paper No. 4, November, 1999. Disponível em SSRN: <http://ssrn.com/abstract=263144> em 21 jun 2007.

SOARES, R. O.; ROSTAGNO, L. M.; SOARES, K. T. C. Estudo de evento: o método e as formas de cálculo do retorno anormal. In: ENCONTRO NACIONAL DOS PROGRAMAS DE PÓS-GRADUAÇÃO EM ADMINISTRAÇÃO, XXVI. Salvador: ANPAD, set. 2002.

STOCKEN, P. C. Credibility of voluntary disclosure. RAND Journal of Economics, vol. 31, summer, p. 359-374, 2000,

WADDOCK, S. A.; GRAVES, S. B. The corporate social performance - Financial performance link. Strategic Management Journal, vol. 18, 4, p. 313-319, 1997.

WATTS, R. L.; ZIMMERMAN, J. L. Positive Accounting Theory: A Ten Year Perspective. The Accounting Review, vol. 65, n. 1, pp. 131-156, January 1990. 
